\title{
THE CORONA CONJECTURE FOR A CLASS OF INFINITELY CONNECTED DOMAINS
}

\author{
BY M. BEHRENS ${ }^{1}$
}

Communicated by Frederick Gehring, June 30, 1969

1. Statement of results. Let $D$ be a domain obtained from the open unit disk $\Delta$ by deleting a sequence of disjoint closed disks $\Delta_{n}$ converging to 0 . We assume that the centers $c_{n}$ and radii $r_{n}$ of the $\Delta_{n}$ satisfy the following two conditions:

$$
\begin{gathered}
\frac{\left|c_{n+1}\right|}{\left|c_{n}\right|} \leqq a<1 \quad \text { for all } n \geqq 1, \text { and } \\
\sum_{n=1}^{\infty} \frac{r_{n}}{\left|c_{n}\right|}<\infty .
\end{gathered}
$$

Let $H^{\infty}(D)$ be the uniform algebra of bounded analytic functions on $D$ and let $\Re\left(H^{\infty}(D)\right)$ be the maximal ideal space of $H^{\infty}(D)$. The Gleason parts of $H^{\infty}(D)$ are the equivalence classes in $\operatorname{mr}\left(H^{\infty}(D)\right)$ defined by the relation $\|\phi-\psi\|<2$, where $\|\cdot\|$ is the norm in the dual of $H^{\infty}(D)$.

With the above assumptions on $D$ we have the following results.

Theorem 1. $D$ is dense in the maximal ideal space of $H^{\infty}(D)$.

THEOREM 2. The Gleason parts of $H^{\infty}(D)$ are all one-point parts or analytic disks, with the exception of the part containing $D$.

The set of homomorphisms $\phi$ of $H^{\infty}(D)$ for which $\phi(z)=0$, where $z$ is the coordinate function on $D$, is called the "fiber over 0 ," and is designated by $\mathfrak{T}_{0}$. $\mathfrak{T}_{0}$ contains the "distinguished homomorphism" $\phi_{0}$ defined by

$$
\phi_{0}(f)=\frac{1}{2 \pi i} \int_{b D} \frac{f(z) d z}{z} .
$$

If $z$ tends to zero in such a way that

$$
\lim _{N \rightarrow \infty}\left(\lim _{z \rightarrow 0} \inf _{n \geqq N} \frac{\left|z-c_{n}\right|}{r_{n}}\right)=\infty
$$

then $f(z)$ tends to $\phi_{0}(f)$ for all $f \in H^{\infty}(D)$, that is, $z$ tends to $\phi_{0}$ in $\mathfrak{N}\left(H^{\infty}(D)\right) . \phi_{0}$ is in the same Gleason part as $D$ (cf. [5]).

${ }_{1}$ The preparation of this paper was supported in part by NSF Grant No. GP-7710. 
Let $N$ be the set of nonnegative integers, $\beta N$ its Cech compactification, and $\widetilde{\beta} N=\beta N \backslash N$.

Theorem 3. The Gleason part containing $D$ is the union of $D$ and a subset $E$ of the fiber over zero. The set $E$ is homeomorphic to the quotient space obtained from $\widetilde{\beta}(N) \times \Delta$ by identifying $\widetilde{\beta}(N) \times\{0\}$ to the point $\phi_{0}$. Each of the functions in $H^{\infty}(D)$ is analytic on each slice of $\widetilde{\beta}(N) \times \Delta$.

The remainder of this note will be devoted to indicating how these theorems are proved, and how they can be extended to more general domains.

2. The algebra $H^{\infty}(\Delta \times N)$. The algebra $H^{\infty}(\Delta \times N)$ of bounded functions on $\Delta \times N$ which are analytic on each slice $\Delta \times\{n\}$ becomes a Banach algebra, when endowed with the supremum norm.

Lemma 1. $\Delta \times N$ is dense in the maximal ideal space $\mathfrak{M}\left(H^{\infty}(\Delta \times N)\right)$ of $H^{\infty}(\Delta \times N)$.

Proof. Suppose $f_{1}, \cdots, f_{n} \in H^{\infty}(\Delta \times N)$ satisfy $\left|f_{1}\right|+\cdots+\left|f_{n}\right|$ $\geqq \delta>0$ on $\Delta \times N$. We must find $g_{1}, \cdots, g_{n} \in H^{\infty}(\Delta \times N)$ satisfying $\sum f_{j} g_{j}=1$. By Carleson's solution of the corona conjecture for the unit disc $\Delta$, there are functions $g_{1 m}, \cdots, g_{n m} \in H^{\infty}(\Delta \times\{m\})$, such that $\sum_{j=1}^{n} f_{j} g_{j m}=1$ on $\Delta \times\{m\}$, and such that $\left|g_{j m}\right| \leqq M$, where $M$ depends only on $\delta$. The $g_{j m}$ then determine functions $g_{j} \in H^{\infty}(\Delta \times N)$ which do the trick.

Now $H^{\infty}(\Delta)$ can be considered a subalgebra of $C(Y)$, where $Y$ is the maximal ideal space of $L^{\infty}(b \Delta, d \theta)$. In fact, $H^{\infty}(\Delta)$ is a strongly logmodular algebra on $Y$, in the sense that every $u \in C_{R}(Y)$ is equal to log $|f|$, for some $f \in H^{\infty}(\Delta)$. Regarding $H^{\infty}(\Delta \times\{m\})$ as a subalgebra of $C(Y \times\{m\})$, we see that $H^{\infty}(\Delta \times N)$ becomes an algebra on the Čech compactification $\beta(Y \times N)$ of $Y \times N$.

Lemma 2. $H^{\infty}(\Delta \times N)$ is a strongly logmodular algebra on $\beta(Y \times N)$.

Proof. Let $u \in C_{R}(\beta(Y \times N))$, and let $u_{m}$ be the restriction of $u$ to $Y \times\{m\}$. There is $f_{m} \in H^{\infty}(\Delta \times\{m\})$ such that $\log \left|f_{m}\right|=u_{m}$, regarded as functions on $Y \times\{m\}$. The $f_{m}$ determine a function $f \in H^{\infty}(\Delta \times N)$ such that $\log |f|=u$ on $Y \times N$, and hence on $\beta(Y \times N)$. That does it.

Now consider the function $Z \in H^{\infty}(\Delta \times N)$, defined by $Z(\lambda, n)=\lambda$. Then $\|Z\|=1$. The Gelfand transform of $Z$ will be denoted by $\hat{Z}$.

Lemma 3. The subset of $\mathfrak{T}\left(H^{\infty}(\Delta \times N)\right)$ on which $|\hat{Z}|<1$ is homeomorphic to $\Delta \times \beta(N)$.

Proof. To each pair $(\lambda, p) \in \Delta \times \beta(N)$ corresponds the homo- 
morphism of $H^{\infty}(\Delta \times N)$ which assigns to $f=\left\{f_{j}\right\}_{j=1}^{\infty} \in H^{\infty}(\Delta \times N)$ the value of the bounded sequence $\left\{f_{j}(\lambda)\right\}_{j=1}^{\infty}$ at $p$. This correspondence is easily seen to be the desired homeomorphism.

Now the closure of each $\Delta \times\{m\}$ is an open subset of $\mathfrak{T}\left(H^{\infty}(\Delta \times N)\right)$. Let $X$ be the space obtained from $\mathscr{T}\left(H^{\infty}(\Delta \times N)\right)$ by deleting the closures of the $\Delta \times\{m\}, m \geqq 1$, and by identifying $\hat{Z}^{-1}(0)$ to a point. Let $A$ be the subalgebra of $C(X)$ obtained by restricting to $X$ the functions in $H^{\infty}(\Delta \times N)$ which are constant on $\hat{Z}^{-1}(0)$. In other words, $A$ is the linear span of $Z H^{\infty}(\Delta \times N)$ and the constants, regarded as continuous functions on $X$.

Lemma 4. $A$ is a uniform algebra on $X$, whose maximal ideal space is $X$. The set $E \subseteq X$ on which $|\hat{Z}|<1$ is homeomorphic to $\Delta \times \widetilde{\beta}(N)$, with $\{0\} \times \widetilde{\beta}(N)$ identified to a point. It forms a Gleason part of $A$. The remaining Gleason parts of $A$ are either points or analytic disks.

Proof. This lemma is easy to verify. The statement concerning the Gleason parts follows from the logmodularity of $H^{\infty}(\Delta \times N)$, and the embedding theorem for analytic disks (cf. [3]).

Note that $E$ is not dense in $X$. In fact, the function $f \in H^{\infty}(\Delta \times N)$, defined by $f(z, n)=z^{n}$, vanishes identically on $E$, while $|f|=1$ on $\beta(Y \times N)$, and hence on the Shilov boundary $\tilde{\beta}(Y \times N)$ of $A$.

3. The isomorphism of the fiber and the fringe. The pairwise disjoint sequence of disks $D_{n}^{c}$ with centers $c_{n}$ and radii $((1-a) / 2) c_{n}$ have the property that every $f \in H^{\infty}\left(\Delta_{n}^{c}\right)$ for which $\|f\| \leqq 1$ satisfies $|f| \leqq(2 /(1-a)) r_{n} /\left|c_{n}\right|$ in $D_{n}$. This, together with condition (ii), gives

Lemma 5. If $\epsilon>0$ and $M>0$ are given, then there exists an integer $Q$ such that: If $f_{n} \in H^{\infty}\left(\Delta_{n}^{c}\right), f_{n}(\infty)=0$, and $\left\|f_{n}\right\| \leqq M$, then

$$
\begin{aligned}
& \sum\left\{\left|f_{m}(z)\right|: m \geqq Q\right\}<\epsilon \quad \text { if } z \in \cup\left\{D_{n}: n \geqq Q\right\}, \\
& \sum\left\{\left|f_{m}(z)\right|: m \geqq Q, m \neq n\right\}<\epsilon \quad \text { if } z \in D_{n} .
\end{aligned}
$$

In particular, $\sum f_{n}$ converges uniformly on compact subsets of $D$ to a function $f \in H^{\infty}(D)$.

For $f \in H^{\infty}(D)$ define

$$
\left(P_{n} f\right)(z)=\frac{1}{2 \pi i} \int_{\partial \Delta_{n}} \frac{f(\xi) d \xi}{\xi-z}, \quad z \in \Delta_{n}^{c} .
$$

Then $P_{n}$ is a projection of $H^{\infty}(D)$ onto the functions in $H^{\infty}\left(\Delta_{n}^{c}\right)$ which vanish at $\infty$. Moreover $P_{j} P_{k}=0$ if $j \neq k$ and $\sup _{n}\left\|P_{n}\right\|<\infty$. If $\left\{f_{n}\right\}$ is 
as in Lemma 5 , then $P_{n}\left(\sum f_{m}\right)=f_{n}$. Applying Lemma 5 to $\left\{P_{n} f\right\}$, we have that $\sum P_{n} f$ converges uniformly on compact subsets of $D$ to $f$ and $f(z) \rightarrow \phi_{0}(f)$ as $z \rightarrow 0, z \in D \backslash \cup D_{n}$. Further,

Lemma 6. Given $\epsilon>0$, there is a $Q$ such that for all $f \in H^{\infty}(D)$ $\left|f(z)-\left(P_{n} f\right)(z)-\phi_{0}(f)\right|<\epsilon$ for $n \geqq Q$ and $z \in D_{n} \backslash \Delta_{n}$.

Let $L_{n}(z)=r_{n} /\left(z-c_{n}\right)$, and define

$$
\Psi(f)(z, n)=\left(P_{n} f\right)\left(L_{n}^{-1}(z)\right)+\phi_{0}(f), \quad f \in H^{\infty}(D) .
$$

Then $\Psi$ is a continuous linear isomorphism of $H^{\infty}(D)$ and those functions in $H^{\infty}(\Delta \times N)$ which are constant on $\hat{Z}^{-1}(0)$. Moreover, if $f \in H^{\infty}(D)$ vanishes on the fiber over 0 , then $\Psi(f)$ vanishes on the "fringe"

$$
\operatorname{m}\left(H^{\infty}(\Delta \times N)\right) \backslash\left(\bigcup_{n=1}^{\infty} \overline{\Delta \times\{n\}}\right) .
$$

Hence $\Psi$ determines a continuous linear operator $\Theta$ from $H^{\infty} / \mathfrak{M}_{0}$ to the algebra $A$ defined in Lemma 4.

Lemma 7. The map $\Theta$ from $H^{\infty}(D) \mid \mathfrak{T \tau}_{0}$ to $A$ is an isometric (algebra) isomorphism.

Proof. By Lemma 6, there is a $Q$ for which

$$
\left|P_{n}(f g)+\phi_{0}(f g)-\left(P_{n} f+\phi_{0}(f)\right)\left(P_{n} g+\phi_{0}(g)\right)\right|<\epsilon
$$

if $n \geqq Q$ and $z \in D_{n} \backslash \Delta_{n}$. Composing with $L_{n}^{-1}$ and using the maximum modulus principle in $\Delta$ we have that

$$
|\Psi(f g)(z, n)-\Psi(f)(z, n) \Psi(g)(z, n)|<\epsilon
$$

for large $n$, and hence that $\Theta$ is multiplicative. That $\Theta$ is isometric follows easily from Lemma 6 , the fact that $f(z) \rightarrow \phi_{0}(f)$ as $z \rightarrow 0$, $z \in D \backslash \cup D_{n}$, and the fact [5] that $\|\hat{f}\|_{\mathfrak{M}_{0}}=\lim \sup _{D \ni z \rightarrow 0}|f(z)|$ for all $f \in H^{\infty}(D)$. As was noted after Lemma $5, P_{n}\left(\sum f_{m}\right)=f_{n}$, so that the image of $\Psi$ covers $Z H^{\infty}(\Delta \times N)$ and hence $\Theta$ is onto $A$.

Proof of Theorem 1. Let $\phi$ be a homomorphism of $H^{\infty}(D)$. If $\phi$ is not in the fiber at 0 then Carleson's corona theorem can be used to show that $\phi$ is in the closure of $D$. Also, $\phi_{0}$ is the closure of $D$. Assume that $\phi \neq \phi_{0}$ is in the fiber over 0. By Lemma $7, \phi$ defines a homomorphism of $A$ and hence a homomorphism $\tilde{\phi}$ of $H^{\infty}(\Delta \times N) . \tilde{\phi}$ is characterized by the fact that $\tilde{\phi}(\Psi(f))=\phi(f)$ for all $f \in H^{\infty}(D)$. Recall that $Z \in H^{\infty}(\Delta \times N)$ is the function defined by $Z(\lambda, n)=\lambda$. For $p \in N$, 
define $I_{p} \in H^{\infty}(\Delta \times N)$ by setting $I_{p}(\lambda, n)=1$ if $n \geqq p$, and $I_{p}(\lambda, n)=0$ if $n<p$. $\tilde{\phi}$ will satisfy $\tilde{\phi}(Z) \neq 0$ and, for each $p \in N, \tilde{\phi}\left(I_{p}\right)=1$.

Let $f_{1}, \cdots, f_{k} \in H^{\infty}(D)$ and $0<\epsilon<|\tilde{\phi}(Z)| / 2$ be given. We will show there is a point $z \in D$ for which $\left|f_{i}(z)-\phi\left(f_{i}\right)\right|<2 \epsilon$ for $i=1, \cdots, k$. Let $p \in N$. By the density of $\Delta \times N$ in $\operatorname{Tr}\left(H^{\infty}(\Delta \times N)\right)$ there is a $(\lambda, n) \in \Delta \times N$ with $\left|\Psi\left(f_{i}\right)(\lambda, n)-\tilde{\phi}\left(\Psi\left(f_{i}\right)\right)\right|<\epsilon$ for $1 \leqq i \leqq k, \mid Z(\lambda, n)$ $-\tilde{\phi}(Z) \mid<\epsilon$ and, $\left|I_{p}(\lambda, n)-\tilde{\phi}\left(I_{p}\right)\right|<\epsilon$. In particular, $|\lambda|>|\tilde{\phi}(Z)| / 2$, and $n \geqq p$. If $p$ was chosen sufficiently large, the last two inequalities guarantee that $L_{n}^{-1}(\lambda) \in D_{n} \backslash \Delta_{n}$. Hence, if $p$ is also larger than the $Q$ of Lemma 6 , then $L_{n}^{-1}(\lambda) \in D$ and

$$
\begin{aligned}
& \left|f_{i}\left(L_{n}^{-1}(\lambda)\right)-\phi\left(f_{i}\right)\right| \\
& \quad=\left|f_{i}\left(L_{n}^{-1}(\lambda)\right)-\left(P_{n} f_{i}\right)\left(L_{n}^{-1}(\lambda)\right)-\phi_{0}\left(f_{i}\right)+\Psi\left(f_{i}\right)(\lambda, n)-\tilde{\phi}\left(\Psi\left(f_{i}\right)\right)\right| \\
& \quad \leqq 2 \epsilon \text { for } i=1, \cdots, k .
\end{aligned}
$$

4. Results for more general domains. These same techniques can be used to prove the following corona theorem.

TheOREM. Let $E$ be a domain for which $E$ is dense in $\operatorname{Tr}\left(H^{\infty}(E)\right)$. Let $D$ be a domain obtained from $E$ by excising a sequence of disjoint closed disks $\Delta\left(c_{n} ; r_{n}\right)$, which satisfy the following conditions:

(i) There exists a disjoint sequence of disks $\Delta\left(c_{n} ; s_{n}\right)$ contained in $E$ with $\sum r_{n} / s_{n}<\infty$,

(ii) $b E$ contains all the limit points of $\left\{c_{n}\right\}$.

Then $D$ is dense in $\operatorname{Tr}\left(H^{\infty}(D)\right)$.

Note that this theorem includes Theorem 1 by taking $E=\Delta \backslash\{0\}$. The proof involves describing the maximal ideal spaces of the fibers of $H^{\infty}(D)$ over $\partial D$. These fibers become immensely more complicated, though, in the general case, than in the simple case we have described.

\section{REFERENCES}

1. L. Carleson, Interpolations by bounded analytic functions and the corona problem, Ann. of Math. (2) 76 (1962), 547-559. MR $25 \# 5186$.

2. T. Gamelin, Uniform algebras, Prentice-Hall, Englewood Cliffs, N. J., 1969.

3. K. Hoffman, Analytic functions and logmodular Banach algebras, Acta Math. 108 (1962), 271-317. MR 26 \#6820.

4. - Banach spaces of analytic functions, Prentice-Hall Series in Modern Analysis, Prentice-Hall, Englewood Cliffs, N. J., 1962. MR 24 \#A2844.

5. L. Zalcman, Bounded analytic functions on domains of infinite connecticity, Trans. Amer. Math. Soc. 144 (1969), 241-269.

University of California, Los Angeles, California 90024 